Objectives: To describe: the pattern of anti-ENA positive tests; frequency of repeated requests; stability and repeatability of anti-Jo- 1 tests; clinical characteristics of anti-Jo-1 + ves compared with controls; and diagnostic value of anti-Jo-1 for ILD.

Methods: All anti-ENA test requests, from any hospital department, between Jan 2013 and Dec 2014 were identified. Serum samples are screened for ENA (Quanta Lite ${ }^{\circledR}$ ENA profile, Inova Diagnostics) and positive samples have specific ENA antibodies levels quantified. Data from anti-Jo-1 positive patients and controls was extracted from electronic records allowing a minimum of 12 months after first test.

Results:

\begin{tabular}{lccc}
\hline & Jo-1 Positive $(\mathrm{n}=40)$ & Controls $(\mathrm{n}=80)$ & $\mathrm{P}$ value \\
\hline Age, mean years (range) & $53(19-86)$ & $52(17-87)$ & - \\
Sex (\% female) & $70 \%$ & $79 \%$ & 0.37 \\
Dead & $13 \%$ & $4 \%$ & 0.12 \\
Current or previous malignancy & $10 \%$ & $10 \%$ & 1.0 \\
Raynauds & $17.5 \%$ & $6.3 \%$ & 0.10 \\
Inflammatory arthritis & $20 \%$ & $19 \%$ & 1.0 \\
Clinical myositis diagnosis & $5 \%$ & $1.3 \%$ & 0.26 \\
CPK $>1000$ units/liter & $5 \%$ & $1.3 \%$ & 0.26 \\
Interstitial lung disease & $12.5 \%$ & $6 \%$ & 0.30 \\
CT chest done during study period & $17 / 40$ & $20 / 80$ & 0.06 \\
ANA ( $\geq 1: 100)$ & $18 / 38(47.4 \%)$ & $22 / 79(27.8 \%)$ & 0.06 \\
RF & $8 / 25(32 \%)$ & $12 / 44(27.3 \%)$ & 0.78 \\
CCP & $0 / 19(0 \%)$ & $3 / 33(9.1 \%)$ & 0.54 \\
Anti-dsDNA (Crithidia +ve) & $7.5 \%$ & $1.3 \%$ & 0.11 \\
Scl70 & $7.5 \%$ & $0 \%$ & $* \star$ \\
SSA Ro & $10 \%$ & $0 \%$ & $* \star$ \\
SSB/La & $10 \%$ & $0 \%$ & ${ }^{* \star}$ \\
RNP & $10 \%$ & $0 \%$ & $* \star$ \\
\hline
\end{tabular}

${ }^{*}$ Fisher's exact test, two tailed. ${ }^{*}$ Statistical analyses were not done on these comparisons as by definition controls were negative for ENA antibodies.

4009 samples from 3581 patients were tested. The first sample tested, chronologically, was designated test of interest. $616(17.2 \%)$ patients were anti-ENA screen +ve, and $40(1.1 \%)$ anti-Jo-1 +ve (>20 AU/mL). Anti-ENA tests were done more than once for $350 / 3581$ (9.8\%) patients (428/4009 (10.7\%) samples) and for $7 / 40(17.5 \%)$ of anti-Jo-1 +ve patients. The median interval between 1st and 2nd requests: 124 days (IQR 233 days). The Table shows data for anti-Jo-1 patients and randomly selected ENA -ve controls. The frequency of ILD, myositis and Raynaud's was comparable. Sensitivity and specificity of Jo-1 for ILD, a key feature of "anti-synthetase syndrome", were $50 \%(\mathrm{Cl} 19-81 \%)$ and $68 \%(\mathrm{Cl} 59-77 \%)$ respectively. Positive predictive value $12.5 \%$ (Cl 4 to $27 \%$ ) and negative predictive value $93.8 \%(\mathrm{Cl} 86-98 \%)$. Of patients with the highest anti-Jo1 titres ( $>40 \mathrm{AU} / \mathrm{mL}, 10 / 40$ patients, 25\%): 3 had ILD, 1 myositis and 2 had a malignancy (disseminated melanoma and CML). Bland-Altman plots show that anti-Jo-1 values remained stable when patients were re-tested at another time but re-testing available stored samples from +ve patients showed important variation (Figure).

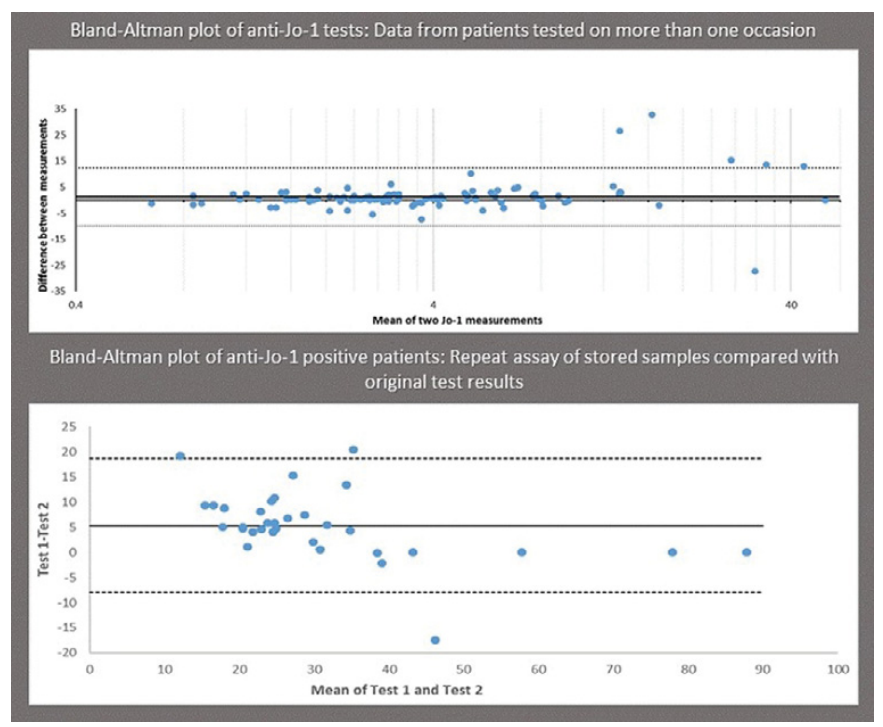

Conclusions: Anti-Jo-1 is uncommon in a heterogenous hospital population and is only weakly predictive for ILD. When tested repeatedly levels remain stable over many months. Repeated testing for anti-ENA is common and potentially unnecessary. Controls over repeated requests could yield cost savings.

Disclosure of Interest: None declared

DOI: 10.1136/annrheumdis-2017-eular.2939

\section{AB1050 COMPARATIVE STUDY BETWEEN ULTRASONOGRAPHIC ASSESSMENT AND CLINICAL EXAMINATION IN RHEUMATOID ARTHRITIS WITH OPTIMIZED BIOLOGICAL THERAPY AND NON BIOLOGICAL TREATMENT}

R. Martínez ${ }^{1}$, M. Fernández ${ }^{1}$, E. Rubio ${ }^{1}$, R. Menor Almagro ${ }^{1}$, B. Hernández ${ }^{2}$, J. Povedano ${ }^{2} .{ }^{1}$ Rheumatology, Hospital Universitario Virgen del Rocío;

${ }^{2}$ Rheumatology, Hospital Universitario Virgen Macarena, Sevilla, Spain

Background: In rheumatoid arthritis (RA), patients in low activity disease or clinical remission measured by disease activity indexs can present subclinical activity by ultrasound study. The ultrasonographic inflammation in examination of joints is an important predictive value of structural damage.

Objectives: The aim of our study was to describe subclinical ultrasonographics activity in patients with RA in low disease activity or clinical remission, with optimized biological therapy and non biological treatment.

Methods: Transversal and longitudinal study describing the ultrasonographic changes in gray scale and doppler in parallel with blind clinical evaluation. We included patients with RA according to ACR/EULAR classification criteria in low activity disease or remission measured by DAS28, under optimized biological therapy at least for 6 months and non biological treatment. They were sent by their usual clinician, making a random selection. They were evaluated in the same day by a rheumatologist and blind sonographer. VAS, VGP, VGM, HAQ, tender joints count, swollen joint count, CDAI, SDAI and DAS28 were evaluated. Regarding the ultrasound, were evaluated synovitis and doppler in 12 joints (wrists, second to fifth MCF and fifth bilateral MTF). The comparison between clinical examination and ultrasonography test was performed by the kappa index, with satisfactory value of $>0,6$

Results: A total of 69 patients were included, 35 with optimized biological therapy and 34 non biological treatment. The median optimization time was 12 months. The baseline characteristics only offered stadistical significance in swollen joint count and average time of disease (table 1).

The concordance study between clinical joint exploration and ultrasonographic examination showed a higher kappa index in patients with optimized biological therapy: 0,52 in gray scale and 0,40 in doppler. In patients without biological therapy showed an index kappa $=0.17$ by the gray scale test, and kappa index $=0.26$ by doppler.

Table 1

\begin{tabular}{lccc}
\hline Variable characteristics & $\begin{array}{c}\text { Non biological } \\
\text { treatment }\end{array}$ & $\begin{array}{c}\text { Optimized biological } \\
\text { therapy }\end{array}$ & $\mathrm{p}$ \\
\hline Age: average $\pm \mathrm{DE}$ (years) & $53,8 \pm 10$ & $54 \pm 11,7$ & 0,834 \\
Women (\%) & 73,5 & 65,7 & 0,498 \\
Time of diseases progression: & $55(34-116)$ & $120(84-139)$ & 0,003 \\
$\quad$ median (p25-p75) (months) & 71,3 & $81,80 \%$ & 0,544 \\
Rheumatoid factor + (\%) & 71,4 & 81,7 & 0,512 \\
Anti CCP + (\%) & $9,0 \pm 1,3$ & $8,1 \pm 1,4$ & 0,658 \\
Metotrexate dose: average \pm DE & $1,7 \pm 0,4$ & $1,5 \pm 0,4$ & 0,856 \\
Corticoids dose: average \pm DE & $1,0 \pm 0,2$ & $1,3 \pm 0,2$ & 0,309 \\
HAQ: average \pm DE & $0(0-1,5)$ & $1(0-2)$ & 0,200 \\
Tender joint count: median (p25-p75) & $0(0-1)$ & $1(0-2)$ & 0,013 \\
Swollen joint count: median (p25-p75) & $7,7 \pm 5,1$ & $6,8 \pm 5$ & 0,437 \\
CDAl: average \pm DE & $10,0 \pm 5,6$ & $10,0 \pm 6,5$ & 0,588 \\
SDAl: average \pm DE & & &
\end{tabular}

Conclusions: Our results show the existing discrepancy between the clinical examination and ultrasonographic test in patients in low disease activity/remission by DAS28, even more with the use of doppler. In the comparison of both groups we observed an increase in the difference in those who did not receive biological therapy. In patients with optimized biological teraphy, with higher swollen joint count in physical examination, kappa index was near of normality in grayscale. The detection of subclinical joint damage is often undertreated, showing ultrasound as a noninvasive technique of great help reducing joint damage.

Disclosure of Interest: None declared

DOI: 10.1136/annrheumdis-2017-eular.4406

\section{AB1051 ULTRASONOGRAPHY AND POWER DOPPLER ULTRASONOGRAPHY OF KNEE JOINT IN PATIENTS WITH HEPATITIS C VIRUS RELATED ARTHRITIS}

R. Elgohary ${ }^{1}$, A. Khalii ${ }^{2}$, G. Ragab ${ }^{3} .{ }^{1}$ Internal Medicine, Rheumatology \& Clinical Immunology subspecialty, Kasr Alainy; ${ }^{2}$ New Kasr El-Aini Teaching Hospital; ${ }^{3}$ Internal Medicine, Rheumatology \& Clinical Immunology subspecialty, Kasr Alainy, Cairo, Egypt

Background: HCV is a hepato-lymphotropic virus. Chronic arthritis is one of its extrahepatic manifestations ${ }^{1}$. The high frequency of rheumatoid factor (RF) positivity in $\mathrm{HCV}$ patients makes it difficult to differentiate between rheumatoid arthritis (RA) and HCV-related arthritis (HCVrA). An accurate and early diagnosis of HCVrA is important to avoid unnecessary immunosuppressive therapy ${ }^{2}$. Ultrasonography provides safe and quick access for the diagnosis of many rheumatic diseases.

Objectives: To illustrate ultrasonographic findings obtained in knee joints of patients with HCVrA \& to compare these findings with those obtained from knee joints of RA patients. 
Methods: Fifteen patients with $\mathrm{HCVrA} \& 15$ RA patients were subjected to: full history, clinical examination, Ultrasonography assessment using a Philips HDI 5000 system with $12-5 \mathrm{MHz}$ Broadband Linear Transducer. Both knees were examined by one ultrasonographer who was blind to clinical data.

Results: In HCVrA, synovial hypertrophy was detected in $10 \%$ of knees $(3 / 30)$ of which $66 \%$ (2 knees) exhibited Doppler signals, while in RA it was detected in 70\% $(21 / 30)$ of which $95 \%$ (20 knees) exhibited Doppler signals. Significant difference was found between the two groups $(p<0.01)$. Knee effusion was detected in $80 \%$ (24/30), \& $86 \%$ (26/30), of patients with HCVrA \& RA respectively, no significant difference was found. Cartilage degeneration was detected in $76 \%(23 / 30)$ \& $83 \%(25 / 30)$, of patients with HCVrA \& RA respectively. Bone erosions were detected in $20 \%(6 / 30)$ in the RA group. It was not detected in HCVrA patients. In $\mathrm{HCV} r \mathrm{~A}$, there was no correlation between the presence of synovial hypertrophy with respect to cartilage degeneration and knee effusion.

Conclusions: We found no specific ultrasonographic feature specific for HCV related knee arthritis, the knee effusion is a predominant feature and the hypertrophied synovium is not frequently found. No destructive lesions were found to be related to the disease itself; however this should be confirmed by histopathological assessment.

\section{References:}

[1] Craxì Antonio, Laffi Giacomo \& Zignego Anna Linda. Hepatitis C virus (HCV) infection: A systemic disease. Molecular Aspects of Medicine 2008;29:85.

[2] Rosner Itzhak, Rozenbaum Michael, Toubi Elias, Kessel Aharon, Naschitz Jochanan, and Zuckerman Eli. The Case for Hepatitis C Arthritis, Seminars in Arthritis and Rheumatism 2004;33:377-379,381-382.

Disclosure of Interest: None declared

DOI: 10.1136/annrheumdis-2017-eular.4094

\section{AB1052 QUALITATIVE SYSTEMATIC REVIEW: LACK OF CONSENSUS ON THE CLASSIFICATION CRITERIA FOR DIFFUSE IDIOPATHIC SKELETAL HYPEROSTOSIS}

J.S. Kuperus ${ }^{1}$, E.E. de Gendt ${ }^{1}$, F.C. Oner ${ }^{1}$, P.A. de Jong ${ }^{2}$, S.C. Buckens ${ }^{2}$, A.E. van der Merwe ${ }^{3}$, G.J. Maat ${ }^{4}$, E.A. Regan ${ }^{5}$, D. Resnick ${ }^{6}$, R. Mader ${ }^{7}$, J.-J. Verlaan ${ }^{1}$. ${ }^{1}$ Orthopedics; ${ }^{2}$ Radiology, University Medical Center Utrecht, Utrecht; ${ }^{3}$ Anatomy, Academic Medical Center, Amsterdam; ${ }^{4}$ Anatomy, Leiden University Medical Center, Leiden, Netherlands; ${ }^{5}$ Medicine, National Jewish Health, Denver, Colorado; ${ }^{6}$ Radiology, University of California San Diego, San Diego, California, United States; ${ }^{7}$ Rheumatology, Ha'Emek Medical Center, Afula, Israel

Background: Diffuse idiopathic skeletal hyperostosis (DISH) is a condition characterized by flowing ossifications of the spine with or without ossifications of entheses elsewhere in the body. ${ }^{1,2}$ Studies on prevalence and pathogenesis of DISH use a variety of partially overlapping combinations of classification criteria, making meaningful comparisons across the literature difficult. ${ }^{3,4}$

Objectives: The aim of this study was to systematically summarize the criteria available to classify or diagnose DISH to aid in the development of a more uniform set of diagnostic and/or classification criteria.

Methods: A search was performed in Pubmed, Embase, Cochrane Library and Web of Science using the term DISH and its synonyms. Articles were included when two independent observers agreed that the articles proposed a new set of classification criteria for DISH. All retrieved articles were evaluated for methodological quality and the presented criteria were extracted. The criteria were placed into one of three groups being "descriptive studies", "sets of criteria for dichotomous diagnosis" or "sets of criteria with consecutive phases".

Results: A total of 24 articles met the inclusion criteria. Two articles were descriptive studies, 11 contained dichotomous classification criteria and 11 described a set of criteria with consecutive phases. In all articles spinal hyperostosis was required for the diagnosis of DISH. Peripheral, extraspinal manifestations were included as a (co-)requirement for the diagnosis DISH in five articles. Most discrepancies revolved around the threshold for the number of vertebral bodies affected and to defining different developmental phases of DISH. More than half of the retrieved articles described a dichotomous set of criteria and did not consider the progressive character of DISH.

Conclusions: In our systematic review we summarize the available different classification criteria for DISH and highlight the lack of consensus on the diagnosis of (early) DISH. Consensus criteria, including consecutive phases of new bone formation that characterize DISH can be developed based upon established diagnostic and/or classification criteria.

\section{References:}

[1] Resnick D, Niwayama G. Radiographic and pathologic features of spinal involvement in diffuse idiopathic skeletal hyperostosis (DISH). Radiology. 1976;119(3):559-568.

[2] Mader R, Verlaan J, Buskila D. Diffuse idiopathic skeletal hyperostosis: clinical features and pathogenic mechanisms. Nat Rev Rheumatol. 2013;9(12):741750.

[3] Pillai S, Littlejohn G. Metabolic factors in diffuse idiopathic skeletal hyperostosis - a review of clinical data. Open Rheumatol J. 2014;8(1):116-128.

[4] Mader R, Buskila D, Verlaan J-J, et al. Developing new classification criteria for diffuse idiopathic skeletal hyperostosis: back to square one. Rheumatology (Oxford). 2013;52(2):326-330.

Acknowledgements: None.
Disclosure of Interest: None declared

DOI: 10.1136/annrheumdis-2017-eular.4656

\section{AB1053 A NOVEL METHOD FOR IDENTIFYING RADIOGRAPHIC BASELINE RISK OF OSTEOARTHRITIS USING AN ANISOTROPY-BASED TEXTURE ANALYSIS ALGORITHM: DATA FROM THE OSTEOARTHRITIS INITIATIVE}

R. Ljuhar $^{1}$, T. Haftner ${ }^{2}$, Z. Bertalan ${ }^{1}$, D. Ljuhar ${ }^{3}$, A. Fahrleitner-Pammer ${ }^{4}$ H.-P. Dimai ${ }^{4}$, S. Nehrer ${ }^{2}$ on behalf of Osteoarthritis. ${ }^{1}$ ImageBiopsy Lab, Vienna; ${ }^{2}$ Department for Health Sciences and Biomedicine, Danube University Krems, Krems; ${ }^{3}$ Braincon Technlogies, Vienna; ${ }^{4}$ Division of Endocrinology and Diabetology, Medical University Graz, Graz, Austria

Background: Osteoarthritis $(\mathrm{OA})$ is the most common form of arthritis and affects disproportionately the knee. Recent developments in imaging techniques showed that $\mathrm{OA}$ is not just a joint disease but also involves progressive changes in the subchondral/subarticular bone area of the tibia. On top of the accepted method of measuring the joint space width, assessments of the trabecular bone structure in selected regions of interest (ROI) in conventional X-rays may be offering an alternative method for quantifying the risk and progression of this disease.

Objectives: The accepted method for assessing OA - Joint space width (JSW) and Joint Space Area (JSA) measurements - have limited capabilities in regard to early identification and reproducible follow-ups of the disease. The objective of this abstract is to evaluate the trabecular bone structure as an area for early identification of OA risk, applying texture anisotropy algorithms and subsequently comparing the results to standard JSW and JSA measurements.

Methods: This study was performed using data from the Osteoarthritis Initiative. The image data set was restricted to female, Caucasian, right knee exams recorded with the same modality. Furthermore we selected exams which had a $\mathrm{KL}$ grade of 0 at the baseline exam and a deteriorating $\mathrm{KL}$ grade $\geq 2$ at the 96 month follow up. 22 cases fulfilled these criteria and we selected 22 matching controls with no signs of $\mathrm{OA}$ at the 96 month follow up. The selected region of interest (ROI) for the analysis of the radiographic texture consisted of four ROls in the subchondral tibia and one additional ROI in each femur condyle - in total 6 ROls. For each individual ROI, the degree of texture anisotropy was calculated and compared between case/control. In addition, JSW \& JSA were calculated in both groups using a proprietary software-based method (ImageBiopsy Lab, Vienna, Austria).

Results: Whereas the JSW and the JSA measurements did not yield any significant differences with respect to their mean values (Cohen's $d=0.139$ and 0.028 ), the calculated texture parameters showed that differences in values between cases and controls can be found in two of the subchondral ROIs (ROI1\&2) with Cohens'd values of 0.625 and 0.831 , respectively. With respect to selected patient, the differences in anisotropy results were significant using these texture parameters.

Conclusions: Our results indicate that using the selected radiographic texture parameters, an early identification of patients at risk for developing OA using conventional X-rays can be achieved. This may offer an additional method for quantifying the risk of baseline OA. This is supported by the Cohen's $d$ values that are by definition relatively large $(0.625$ and 0.831$)$. Ongoing research focuses on larger sample set validation and the use of such algorithms for additional applications, such as the early identification of fracture risk.

Disclosure of Interest: None declared

DOI: 10.1136/annrheumdis-2017-eular.5860

\section{AB1054 QUANTITATIVE ULTRASOUND FOR ASSESSMENT OF DISEASE ACTIVITY IN PATIENTS WITH RHEUMATOID ARTHRITIS: RELIABILITY AND CONSTRUCT VALIDITY}

R.P. Goswami, A. Ghosh, D. Lahiri, P. Ghosh. Department of Rheumatology, IPGMER, KOLKATA, INDIA, Institute of Post Graduate Medical Education and Research, Kolkata, India

Background: Joint ultrasound is an integral part of assessment of patients with rheumatoid arthritis (RA). Semi-quantitative grading of ultrasound is subjective whereas quantitative ultrasound (QUS) may be more objective.

Objectives: To evaluate the reliability and construct validity of QUS of wrist joints in patients with RA.

Methods: We studied 95 patients with RA. Following parameters were studied: swollen and tender joint counts (SJC and TJC), patient and evaluator global assessment (PGA, EGA) and disease activity score (DAS28). Patients were classified as active disease and disease in remission as per expert clinician opinion. Colour Doppler ultrasound (CDUS) of wrists was done to obtain semiquantitative grading. Scans were processed in image analysis software (Photoshop CS4) to obtain the following: colour fraction of intrasynovial (IS) vascular signals $(C F(I S)=$ pixel area of IS vascular signals/pixel area of entire IS area (area(IS)), CF(total) (pixel area of both IS and extrasynovial vascular signals/area (IS)) and number of IS (N(IS)) and extrasynovial (N(ES)) vascular signals. Images were stored and independently rated for both CDUS and QUS by two different raters blinded to each other's rating.

Results: Demographics of patients were: mean age $48 \pm 16.7$ years, mean disease duration 24 months (range $4-600)$, mean DAS28 of $2.98 \pm 1.18$ and $40 \%(38 / 95)$ 